\title{
The use of chemosensitizers to enhance the response to conventional therapy in triple-negative breast cancer patients
}

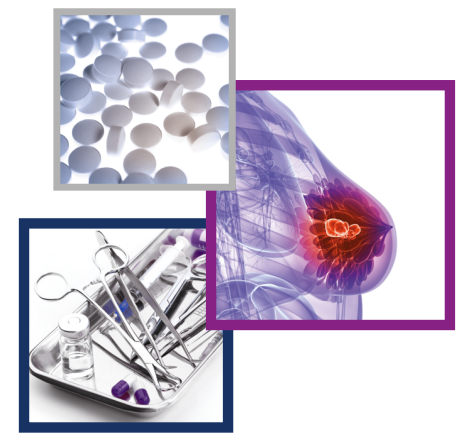

\author{
Fouzia Guestini*,1, Keely May McNamara \& Hironobu Sasano ${ }^{1}$ \\ ${ }^{1}$ Department of Anatomic Pathology, Graduate School of Medicine, Tohoku University, Sendai 980-8575, Japan \\ * Author for correspondence: guestini.fouzia@gmail.com
}

\begin{abstract}
"Therefore, phytochemicals are generally considered pharmacologically safe natural products capable of downregulating the effectors of pathways involved in multidrug resistance and thereby circumventing chemotherapeutic drug resistance and also enhance their antiproliferative effects."
\end{abstract}

First draft submitted: 16 December 2017; Accepted for publication: 22 December 2017; Published online: 2 February 2018

Keywords: chemotherapy $\bullet$ combined treatment $\bullet$ radiotherapy $\bullet$ sensitization $\bullet$ triple-negative breast cancer

Chemotherapy is the standard of care in triple-negative breast cancer (TNBC) treatment, combining cytotoxic drugs consisting of anthracyclines, taxanes, cyclophosphamide and platinum, all of which could induce cell death through various mechanisms. However, concomitant adverse side effects and development of multidrug resistance has led to the search for new drugs or combinatory drug regimen [1]. Thus, any compounds which can hamper tumor survival signals could be considered as chemosensitizer and used to enhance the efficacy of standard chemotherapy. However, considering the individual biopharmaceutical properties of the chemosensitizers, achieving a stable coload of reactive payloads of chemotherapeutic agents and chemosensitizers and avoiding drug leakage during in vivo transportation constitute enormous challenges to overcome for the approval of this novel therapy at this juncture. In addition, the emergence of multidrug resistance of carcinoma cells against multiple unrelated drugs, due to drug efflux from the tumor cell involving the ATP-binding cassette transporter superfamily, could result in increased doses of effective drugs in actual clinical practice. Therefore, chemosensitizers with the ability to accumulate preferably within tumor site and potentialize systemic conventional therapeutic effects could represent potential candidates [2-4]. In this study, we will review the most current literature to shed a light upon pivotal experimental approaches and landmark studies that have charted strategies to overcome resistance to standard therapy in TNBC patients. We will put our focus upon natural products, agents facilitating the effects of DNA damaging drugs and genomic approaches as chemosensitizers.

Despite the proven efficiency of synthetic chemicals for cancer treatment, clinical application of natural products is still appreciated, due to low systemic toxicity, in addition to the fact that they also exert antitumor effects and could act as potent chemosensitizers in combination with conventional chemotherapeutic drugs. Moreover, they can increase drug efficacy at lower dose levels thus, reducing dosage toxicity and drug resistance [5-7]. Recently, several studies have reported the ability of phytochemicals such as flavonoids, alkaloids and polyphenols to sensitize TNBC cells to chemotherapeutics and reverse multidrug resistance. To mention but a few, the use of rutin that enhanced the cytotoxicity of cyclophosphamide and methotrexate on TNBC cells (MDA-MB-231), ER- and PR-positive breast cancer cells (MCF-7) and primary human mammary fibroblasts in a time dependent manner, through decreasing the activities of the ATP-binding cassette transporters, P-gp and BCRP, cell cycle arrest at G2/M and G0/G1 phases and promoting cell apoptosis [7]. An additional example would be noscapine, a drug commonly used as a cough suppressant with anticancer properties on different cancer types, sharing a common cellular target (microtubules) as doxorubicin, synergistically increased the cytotoxicity of the chemo-drug via inactivation of NF$\kappa \mathrm{B}$ and antiangiogenic pathways while stimulating apoptosis against TNBC tumors. In addition, the combined treatment led to the activation of early stress markers such as P38 and JNK and downregulation of p-Akt, Bcl-2 and 
survivin, consequently inducing apoptosis in doxorubicin-treated breast cancer cells and the decrease of $\alpha$-tubulin, required for microtubules formation. In vivo, noscapine reduced collagen-I in TNBC solid tumor and also increased the intratumoral uptake of coumarin-6 loaded polyethylene glycoylated liposomes, providing the rationale of its ability to increase the bioavailability of nanocarriers loaded with anticancer drugs in solid human tumors [6]. Curcumin has exhibited inhibitory effects on several malignancies including breast cancer. Due to its pleiotropic effect; curcumin has been reported to regulate various intracellular signaling pathways and genes in different breast carcinoma cell lines [8]. Curcumin downregulated the FABP5 $/ \mathrm{PPAR} \beta / \delta$ pathway and restored the sensitivity of retinoid acid-resistant TNBC cells to the inhibitory effect of retinoid acid drugs [5]. Curcumin synergistically chemo-sensitized breast cancer cells to 5 -fluorouracil, reducing the drug toxicity and resistance through thymidylate synthase-dependent downregulation of NF- $\mathrm{B}$, thus leading to enhancement of apoptosis [9]. In another study, the combination of curcumin with mitromycin was reported to reduce drug induced side effects, by inhibiting GRP58mediated DNA crosslinking through ERK/p38 MAPK pathway [10]. Guo et al. suggested a novel self-assembled polycurcumin nanoparticle used both as a pre-chemosensitizer and as a drug carrier (doxorubicin) for an effective treatment of multidrug resistance breast cancer in vivo. The self-assembled nanoparticle had a high loading capacity of both hydrophobic multidrugs, was capable of co-releasing the chemosensitizer that could enhance tumor cell death and co-delivering the encapsulated chemotherapeutic drug doxorubicin specifically within the tumor in the multidrug-resistant MCF-7/ADR xenografted nude mice [4]. This new technology provides the advantage of an intracellular co-release of drugs including chemotherapeutic drug and chemosensitizer to maximize the antitumor effect against multidrug resistant cancers. Therefore, phytochemicals are generally considered pharmacologically safe natural products capable of downregulating the effectors of pathways involved in multidrug resistance and thereby circumventing chemotherapeutic drug resistance and also enhance their antiproliferative effects. Nevertheless, methods of enhancing their bioavailability in vivo still require further investigations.

The tumor suppressors BRCA1/2 proteins are well known to be responsible for both DNA damage sensing and the homologous recombination (HR) dependent DNA-repair pathways. A relatively high incidence of germline mutations in the BRCA1/2 genes was reported in TNBCs patients which makes them more sensitive to DNA damaging agents such as platinum and PARP inhibitors, the last of which are particularly active in tumors with defects in DNA HR [1]. Studies provided evidence that targeting key signaling pathways could potentialize the therapeutic effects of the DNA damaging drugs [11-13]. The PI3K signaling pathway preserves HR steady state, thus the inhibition of PI3K pathway would result in HR impairment in BRCA-proficient TNBC patients and subsequently sensitize the carcinoma cells to PARP inhibitors. This was clearly demonstrated by Ibrahim et al. who showed that the inhibition of PI3K resulted in the downregulation of BRCA1/2, DNA damage, gain in polyADP-ribosylation and subsequent sensitization to PARP inhibition in TNBC cell model. The inhibition of PI3K was indeed accompanied by the overexpression and subsequent activation of MEK1 and ERK phosphorylation respectively, with the involvement of ETS1 transcription factor in the downregulation of BRCA1/2 and the accumulation of phosphorylated $\gamma \mathrm{H} 2 \mathrm{AX}$, a marker of DNA double-strand breaks. These results provided the rationale of targeting PI3K pathway in order to potentialize the effects of PARP inhibitors in TNBC patients regardless of their BRCA1/2 germline mutation profile [11]. A Phase I clinical trial combining oral PI3K inhibitors with the PARP inhibitor olaparib is ongoing (NCT01623349). Another recent study unveiled the involvement of an alternative pathway to PI3K, which caused a defect in the HR DNA repairing pathway and sensitized TNBC cell lines to PARP inhibition. The results shed light on the role played by the TAM family receptor tyrosine kinase AXL in epithelial-mesenchymal transition and, for the first time, HR DNA repair pathway, suggesting the use of AXL inhibitors as chemosensitizers in combination with PARP inhibitors in breast cancer [13]. Breast cancer patients with impairment in DNA damage response, HR proteins and BRCA1/2 all harbor a relatively high genomic instability and defective DNA repair machinery; subsequently, they became sensitive to DNA damaging agents such as, PARP inhibitors and platinum agents. Findings reported by $\mathrm{Ha}$ et al. did demonstrate that treatment of TNBC cells with pan-HDAC inhibitors created an imbalance in pro-survival and antiapoptotic proteins in favor of pro-death members, induced hyperacetylation of HSP90 and disrupted its chaperone function, causing impairment in DNA damage response (depletion of ATR and CHK1) and HR (depletion of BRCA1 and RAD51), thus synergistically sensitizing TNBC cells in vitro and in vivo to DNA damaging agents PARP inhibitors or cisplatin [12]. An interesting recent finding addressed the use of $\mathrm{CHK} 1$ inhibitors as radiotherapy potentiators. The pretreatment with the drug led to the inhibition of irradiation-induced autophagy and enhanced DNA damage in TNBC cell lines (MDA-MB231, BT-549 and CAL-51) and in MDA-MB-231 xenograft tumors nude mice [14]. Collectively, the supportive findings above made a strong case for the rationale of enhancing DNA damage to potentialize chemotherapy or 
radiotherapy through the combination of PI3K inhibitors, AXL inhibitors and pan-HDAC inhibitors with DNA damaging drugs in TNBC irrespective of BRCA1 expression or the use of CHK1 inhibitors prior to radiotherapy.

Genomic profiling of tumors has generally provided useful insights into mechanisms of tumorigenesis offering important clinically relevant information. Various genomic techniques have been used to ensure specific inhibition of tumor cell growth or identify modulators of drug sensitivity, toward enhancing drug sensitivity and improving the response rate with subsequent reduction of therapeutic resistance $[2,15,16]$. Among those above, first, antisense oligonucleotides (ODN), which could specifically inhibit the synthesis of a particular cancer-associated protein by binding to protein-encoding RNA, thereby preventing RNA function [15]. Second, RNAi using synthetic siRNAs or vector-based shRNAs, specifically knockdown the expression of target genes via DICER and RNA-induced silencing complex, thus leading to the degradation of the mRNA and preventing the expression of the corresponding protein [2]. Moreover, this last approach could uncover novel gene functions and unveil novel pathways, offering perspectives to identify drug combinations and find new molecules therapeutically targetable in various tumor types [16]. The Bcl-2 family comprises pro-survival antiapoptotic proteins (Bcl-2, Bcl-xL, Mcl-1, Bcl-w and A-1) and pro-apoptotic proteins (Bax, Bak, Bik, Bad, Bid, HRK, BMF, NOXA and PUMA). The properties of Bcl-2 extend from the regulation of apoptosis and autophagy to the promotion of cancer progression by enhancing cell invasion, cell migration and metastasis which is partly responsible of the biologically aggressiveness of TNBCs overexpressing the oncogene and developing resistance to conventional therapies. Therefore, the candidates above could represent a promising target for molecular intervention strategies to inhibit their expression. The evidence of the cytoprotective function of $\mathrm{Bcl}-2$ and $\mathrm{Bcl}-\mathrm{xL}$ and their overexpression in breast cancer highlighted the evidence of the efficiency of ODN targeting the apoptotic pathways to sensitize breast carcinoma cells to standard anticancer therapy $[15,17]$. An interesting finding of Nahta et al. shed light on the potential contribution of the induction of BTG1 in the cytotoxicity mediated by antisense Bcl-2 on ER-positive MCF-7 and negative MDA-MB-231 breast cancer cells, suggesting BTG1 as a novel Bcl-2-regulated mediator of apoptosis in breast carcinoma cells, whose induction contributes to antisense Bcl-2-mediated cell death [18]. The in vitro and in vivo studies of the effects of transient $\mathrm{Bcl}-2$ downregulation revealed the promising potential of using Bcl-2 antisense ODN as a combinatory treatment for breast cancer. Of particular interest, in vitro use of antisense approach facilitated tumor-cell apoptosis and reduced the dose of standard anti-cancer drugs required to achieve clinically significant anti-tumor responses [19]. In another study, the transfection of ER-positive or ER-negative breast cancer cell lines with Bcl-2 antisense ODN were also reported to result in a stable decrease of Bcl-2 expression at the mRNA and protein level [20] and cell death, exhibiting properties indicative of apoptosis such as mitochondrial membrane depolarization and caspase activation [20,21]. In the in vivo model, a shortly reduced expression of the $\mathrm{Bcl}-2$ protein was also reported, followed by a resistance to continued $\mathrm{Bcl}-2$ ODN treatment [17]. Interestingly, a combination of treatment with $\mathrm{Bcl}-2$ antisense ODN and cytotoxic agents such as 5-fluorouracil [20], free doxorubicin or sterically stabilized liposomal doxorubicin [17], all resulted in additive cytotoxicity or synergistic in both breast cancer cell lines type [20,21] and breast-solid tumor xenograft model, enhanced when combined with liposomal formulations of anticancer drugs [17]. In addition, Simões-Wüst $e$ t al. reported a simultaneous downregulation of $\mathrm{Bcl}-2$ and $\mathrm{Bcl}-\mathrm{xL}$ by the use of the bispecific antisense ODN 4625 that synergistically sensitized cells to chemotherapy (doxorubicin, paclitaxel or cyclophosphamide), inducing apoptosis via the mitochondrial pathway which is controlled by Bcl-2 and Bcl-xL. This was mainly characterized in the case of paclitaxel, an agent that influences the stability of microtubule spindles, which led to mitotic catastrophe with cells arrested in mitosis evolving to a multinucleated state [19]. In a recent study, the authors used the same antisense gene target approach (antisense miR-21 nanoparticles) in combination with engineered nanoparticles loaded with the antiobesity drug Orlistat. The results did demonstrate an increased bioavailability of the nanoparticle-loaded-drug, which enhanced the therapeutic effect of the chemotherapeutic agents on both MDA-MB-231 and SkBr3 cells in vitro and increased antiapoptotic synergistic effect of the combined drugs compared with the independent use of the drugs. Same results were observed when combining Orlistat and doxorubicin [3].

A novel promising approach that allows the knockdown of sequence-specific genes involved in drug resistance pathways using RNAi mediated by siRNA enabled targeting Bcl-2 in breast cancer; nevertheless some challenges remain as how to deliver the drugs into the tumor in vivo, due to a poor stability and diffusion into the cancer cells [2]. Two groups have reported the advantage of $\mathrm{Bcl}-2$ silencing therapy in breast carcinoma cells. The first study by Tekedereli et al. demonstrated that the systemic administration of Bcl-2 siRNA loaded in nanoliposomes in MDA-MB-231 and MCF7 breast tumors in orthotopic xenograft provided robust and persistent knockdown of the target gene expression. In addition, it inhibited tumor growth in both cell animal models and also enhanced 
the efficacy of the chemotherapy when co-administered with doxorubicin. Interestingly, this study demonstrated that $B c l-2$ silencing, using this approach, reduced the activity of FAK/SRC, HIF $1 \alpha$ and cyclin, all known to play pivotal roles in cell migration, cell invasion and metastasis [2]. A more recent work reported the construction of an efficient, stable colloidal formulation of hydrophobically associated multiple monocomplex composed of siRNA targeting the oncogene $B c l-2$, an amonocationic surfactant with anticancer activity (benzethonium chloride, BZT), and a biocompatible polymeric surfactant (pluronic F-68) for micelle encapsulation. This ternary complex successfully co-delivered the siRNA and BZT into the tumor cytoplasm after a peritumoral administration in a TNBC resistant mice model, downregulated Bcl-2 expression and thereby enabled BZT-induced apoptosis. This same chemo-sensitizing effect with BZT was also reported when replaced by doxorubicin hydrochloride.

These data all pointed out the potential of the gene-targeted combination therapy in clinical use for locoregional cancer treatment [22]. Finally, a novel approach of gene silencing on TNBC, a holliday junction structure combined a cell targeting aptamer and three siRNA strands targeting Akt, MDM2 and survivin in MDA-MB-231 TNBC cell line. The results demonstrated a downregulation of the three gene targets, an upregulation of phospho-p 53 which subsequently led to reduced cell survival within $24 \mathrm{~h}$ at a very low dose. This particular four-arm nanoconstruct of the holiday junction was reported to represent a potential contender for designing gene antisense therapy [23]. These studies suggested that therapeutics using genomic techniques including miRNA or RNAi, targeting key genes involved in drug resistance, combined to nanocarrier particles are viable approaches in the treatment of breast cancer patients.

In conclusion, targeting key effectors regulating multidrug resistance pathways does constitute an attractive therapeutic approach. In addition, a combinatory treatment of chemotherapeutic agents with natural products, targeted therapy or genomic technology to enhance the antitumoral effect of conventional drugs represent the forefront strategy to circumvent TNBC drug resistance. Nevertheless, further investigation on drug bioavailability, stability and specific reactivity of the agents on TNBC cells in vitro and in vivo are required to verify their potential as adjuvant agent in TNBC therapy and avoid the torment of the development of a resistance to new drugs.

Financial \& competing interests disclosure

The authors have no relevant affiliations or financial involvement with any organization or entity with a financial interest in or financial conflict with the subject matter or materials discussed in the manuscript. This includes employment, consultancies, honoraria, stock ownership or options, expert testimony, grants or patents received or pending, or royalties.

No writing assistance was utilized in the production of this manuscript.

\section{References}

1. Guestini F, McNamara KM, Ishida T, Sasano H. Triple-negative breast cancer chemosensitivity and chemoresistance: current advances in biomarkers indentification. Expert Opin. Therapeut. Targets 20(6), 705-720 (2016).

2. Tekedereli I, Alpay SN, Akar U et al. Therapeutic silencing of Bcl-2 by systemically administered siRNA nanotherapeutics inhibits tumor growth by autophagy and apoptosis and enhances the efficacy of chemotherapy in orthotopic xenograft models of ER (-) and ER (+) breast cancer. Mol. Ther. Nucleic Acids 2, e121 (2013).

3. Bhargava-Shah A, Foygel K, Devulapally R, Paulmurugan R. Orlistat and antisense-miRNA-loaded PLGA-PEG nanoparticles for enhanced triple-negative breast cancer therapy. Nanomedicine 11(3), 235-247 (2016).

4. Guo S, Lv L, Shen Y, Hu Z, He Q, Chen X. A nanoparticulate pre-chemosensitizer for efficacious chemotherapy of multidrug resistant breast cancer. Sci. Rep. 6, 21459 (2016).

5. Thulasiraman P, McAndrews DJ, Mohiudddin IQ. Curcumin restores sensitivity to retinoic acid in triple-negative breast cancer cells. BMC Cancer 14(1), 724 (2014).

6. Doddapaneni R, Patel K, Chowdhury N, Singh M. Noscapine chemosensitization enhances docetaxel anticancer activity and nanocarrier uptake in triple-negative breast cancer. Exp. Cell Res. 346(1), 65-73 (2016).

7. Iriti M, Kubina R, Cochis A et al. Rutin, a quercetin glycoside, restores chemosensitivity in human breast cancer cells. Phytother. Res. 31(10), 1529-1538 (2017).

8. Wang Y, Yu J, Cui R, Lin J, Ding X. Curcumin in treating breast cancer: a review. J. Lab. Auto. 21(6), 723-731 (2016).

9. Vinod BS, Antony J, Nair HH et al. Mechanistic evaluation of the signaling events regulating curcumin-mediated chemosensitization of breast cancer cells to 5-fluorouracil. Cell Death Dis. 4(2), e505 (2013).

10. Zhou QM, Wang XF, Liu XJ et al. Curcumin improves MMC-based chemotherapy by simultaneously sensitising cancer cells to MMC and reducing MMC-associated side-effects. Eur. J. Cancer 47(14), 2240-2247 (2011). 
11. Ibrahim YH, Garcia-Garcia C, Serra V et al. PI3K inhibition impairs BRCA1/2 expression and sensitizes BRCA-proficient triple-negative breast cancer to PARP inhibition. Cancer Discov. 2(11), 1036-1047 (2012).

12. Ha K, Fiskus W, Choi DS, Bhaskara S, Cerchietti L. Histone deacetylase inhibitor treatment induces 'BRCAness' and synergistic lethality with PARP inhibitor and cisplatin against human triple-negative breast cancer cells. Oncotarget 5(14), 5637-5650 (2014).

13. Balaji K, Vijayaraghavan S, Diao L et al. AXL inhibition suppresses the DNA damage response and sensitizes cells to PARP inhibition in multiple cancers. Mol. Cancer Res. 15(1), 45-58 (2017).

14. Zhou ZR, Yang ZZ, Wang SJ, Zhang L, Luo JR, Feng Y. The Chk1 inhibitor MK-8776 increases the radiosensitivity of human triple-negative breast cancer by inhibiting autophagy. Acta Pharmacol. Sin. 38(4), 513-523 (2017).

15. Biroccio A, Leonetti C, Zupi G. The future of antisense therapy: combination with anticancer treatments. Oncogene 22(42), 6579-6588 (2003).

16. Bauer JA, Fei Y, Clayton M et al. RNA interference (RNAi) screening approach identifies agents that enhance paclitaxel activity in breast cancer cells. Breast Cancer Res. 12(3), R41 (2010).

17. Lopes de Menezes DE, Hudon N, McIntosh N, Mayer LD. Molecular and pharmacokinetic properties associated with the therapeutics of Bcl-2 antisense oligonucleotide G3139 combined with free and liposomal doxorubicin. Clin. Cancer Res. 6(7), 2891-2902 (2000).

18. Nahta R, Yuan LXH, Fiterman DJ et al. B cell translocation gene 1 contributes to antisense $B c l$-2-mediated apoptosis in breast cancer cells. Mol. Cancer Ther. 5(6), 1593-1601 (2006).

19. Simōes-Wüst AP, Schürpf T, Hall J, Stahel RA, Zangemeister-Wittke U. Bcl-2/bcl-xL bispecific antisense treatment sensitizes breast carcinoma cells to doxorubicin, paclitaxel and cyclophosphamide. Breast Cancer Res. Treat. 76, 157-166 (2002).

20. Yang JH, Feng F, Qian H, Cheng H. Chemosensitization of breast carcinoma cells with the use of $b c l-2$ antisense oligodeoxynucleotide. Breast 13(3), 227-231 (2004).

21. Chi KC, Wallis AE, Lee $\mathrm{CH}$ et al. Effects of Bcl-2 modulation with G3139 antisense oligonucleotide on human breast cancer cells are independent of inherent Bcl-2 protein expression. Breast Cancer Res. Treat. 63(3), 199-212 (2000).

22. Lee E, Oh C, Kim IS, Kwon IC, Kim S. Co-delivery of chemosensitizing siRNA and an anticancer agent via multiple monocomplexation-induced hydrophobic association. J. Control Release 210, 105-114 (2015).

23. Tung J, Tew LS, Hsu YM, Khung YL. A novel 4-arm DNA/RNA nanoconstruct triggering rapid apoptosis of triple-negative breast cancer cells within 24 hours. Sci. Rep. 7(1), 793 (2017). 